\title{
Papel da 2-tridecanona e dos tricomas glandulares tipo VI na resistência do tomateiro a Tuta absoluta ${ }^{(1)}$
}

\author{
Elsa Gilardón ${ }^{(2)}$, Mariana Pocovi(2), Carmen Hernández ${ }^{(2)}$, Graciela Collavino ${ }^{(2)}$ e Ana Olsen ${ }^{(2)}$
}

\begin{abstract}
Resumo - O metabólito secundário 2-tridecanona, secretado pelos tricomas glandulares tipo VI das folhas de tomateiro silvestre, Lycopersicon hirsutum L., confere-lhe resistência a uma grande variedade de insetos, inclusive a traça-do-tomateiro, Tuta absoluta (Meyrick). O objetivo do trabalho foi avaliar a concentração de 2- tridecanona, a densidade de tricomas glandulares tipo VI e o grau de infestação da traça-do-tomateiro na cultivar suscetível 'Uco Plata' (Lycopersicon esculentum Mill.), na linhagem resistente PI 134417 (L. hirsutum f. glabratum) e em suas progênies $\mathrm{F}_{1}$ e $\mathrm{F}_{2}$. Foram avaliadas ainda as possíveis associações entre a concentração de 2-tridecanona, a densidade de tricomas glandulares tipo VI e o grau de infestação da traça-do-tomateiro. O grau médio de infestação da traça-do-tomateiro em 'Uco Plata' foi significativamente superior ao obtido em PI 134417. Ainda que a concentração de 2-tridecanona tenha sido significativamente superior no parental resistente, a presença deste metabólito somente explicaria parcialmente a resistência $\left(\mathrm{R}^{2}=8,17 \%\right)$. Não se detectaram diferenças significativas na densidade de tricomas tipo VI entre o parental suscetível e o resistente. Este comportamento ocorreu independentemente do conteúdo de 2-tridecanona e do grau de infestação da traça-do-tomateiro.
\end{abstract}

Termos para indexação: Lycopersicon hirsutum, herbívoros, infestação, metabólicos, resistência a pragas.

Role of 2-tridecanone and type VI glandular trichome on tomato resistance to Tuta absoluta

Abstract - The secondary metabolite 2-tridecanone, secreted by the type VI glandular trichomes present in the leaves of Lycopersicon hirsutum L., make it resistant to a great variety of insects, including the tomato pinworm, Tuta absoluta (Meyrick). The 2-tridecanone concentration, the type VI glandular trichome densities and the pinworm infestation degree were evaluated on the Lycopersicon esculentum Mill. susceptible cultivar 'Uco Plata', on the Lycopersicon hirsutum f. glabratum resistant accession PI 134417 and on their $F_{1}$ and $F_{2}$. The mean infestation degree with pinworm larvae was significantly higher in 'Uco Plata' than in PI 134417. The 2-tridecanone concentration was significantly higher in the resistant parent, but the presence of this metabolite could only partially account for the resistance $\left(\mathrm{R}^{2}=8.17 \%\right)$. No significant differences were detected in the type VI glandular trichomes densities between the susceptible and resistant parents. This trait was independent of the 2-tridecanone level and the pinworm infestation degree.

Index terms: Lycopersicon hirsutum, herbivores, infestation, metabolites, pest resistance.

\section{Introdução}

Numerosos estudos citam que a resistência bioquímica é o mecanismo de defesa mais importante que as plantas desenvolvem contra o ataque dos herbívoros. Muitos compostos químicos podem ser a causa da resistência, incluindo compostos inorgânicos, metabólitos primários e secundários (Maxwell \& Jennings, 1980).

\footnotetext{
(1) Aceito para publicação em 14 de junho de 2000 .

(2) Universidad Nacional de Salta. Buenos Aires 177, 4400

Salta, Argentina. E-mail: gilardon@unsa.edu.ar
}

A distinção entre um metabólito primário e um secundário não é totalmente clara (Rosenthal \& Berenbaum, 1992). Alguns autores reservam o termo metabólito secundário ou aleloquímico para referirse exclusivamente aos produtos secundários biossintetizados pelas plantas (Wink, 1988). Sua distribuição está restrita aos grupos de organismos taxonomicamente relacionados. Geralmente conferem cor, cheiro ou sabor característico ao indivíduo que os produz. Suas funções estão relacionadas com a sobrevivência da espécie: a toxicidade e repelência, como mecanismo de defesa (contra herbívoros, predadores e microrganismos) e a atração de polinizadores (Maxwell \& Jennings, 1980). 
Durante a domesticação dos cultivos, foi se modificando o 'pool' gênico das plantas silvestres (Wink, 1988). As mutações, recombinações e outras alterações levaram a mudanças qualitativas e quantitativas na produção de aleloquímicos. Muitos destes compostos perderam-se e com eles a capacidade das plantas de se defender (Luckner, 1989). Por exemplo, as espécies de tomate silvestre, similares às cultivadas, conservam os genes envolvidos na biossíntese de potentes defesas químicas que lhes conferem resistência a uma grande variedade de insetos. Lycopersicon hirsutum f. glabratum tem sido classificado como altamente resistente ao ataque de numerosos insetos, entre eles Tuta absoluta (Saravia et al., 1984; Gray et al., 1999), Manduca sexta (Kennedy et al., 1981; Fery \& Kennedy, 1987), Phtorimaea operculella, Spodoptera exigua e Heliothis armigera (Juvik \& Stevens, 1982).

Uma das principais pragas do tomate cultivado é a traça-do-tomateiro, Tuta absoluta Meyr. (Lepidoptera: Gelechiidae). Este inseto tem distribuição neotropical e constitui uma das pragas mais nocivas da América do Sul (Maluf et al., 1997). O adulto oviposita sobre as folhas do tomate e suas larvas fazem sulcos no mesófilo das folhas, nos ramos, nas flores e nos frutos, diminuindo-lhes significativamente a qualidade. Seu controle normalmente é feito com inseticidas, os quais podem contaminar o ambiente e deixar, nos frutos, resíduos tóxicos para o homem.

Um dos metabólitos secundários presentes nos tricomas glandulares tipo VI das folhas do tomate é a metilcetona 2-tridecanona. Constitui $61 \%$ dos componentes oleosos das folhas de L. hirsutum e 30\% dos de L. esculentum (Fery \& Kennedy, 1987). É o principal produto natural responsável pela resistência aos insetos Manduca sexta, Heliothis zea (Williams et al., 1980), Leptinotarsa decemlineata (Kennedy et al., 1991) e Spodoptera exigua (Eigenbrode \& Trumble, 1993). Giustolin \& Vendramim (1996) informaram que a adição de 2-tridecanona em diferentes concentrações a uma dieta artificial de T. absoluta provoca $100 \%$ de mortalidade das larvas. Maluf et al. (1997), trabalhando com um cruzamento entre $L$. esculentum TSWV-547 e L. hirsutum f. glabratum (PI 134417), entenderam ser 2-tridecanona o principal metabólito envolvido na resistência a T. absoluta, atuando como mecanismo de repelência às larvas e na oviposição dos adultos. No entanto, Gray et al. (1999), utilizando o método colorimétrico de quantificação da 2-tridecanona, detectaram só uma leve, porém significativa associação negativa entre o nível deste composto e o grau de infestação da traça-do-tomateiro, concluindo que este metabólito não seria o principal componente químico da resistência a essa praga.

Os tricomas glandulares tipo VI secretam também outros metabólitos como os fenóis, rutina e ácido clorogênico (Juvik et al., 1988), lipídios e o sesquiterpeno zingibereno (Snyder \& Carter, 1984). Esses dois autores ainda mencionam a relação entre a densidade de tricomas glandulares tipo VI e a resistência a Tetranychus urticae Koch.

Os objetivos deste trabalho foram avaliar o nível de 2-tridecanona, a densidade de tricomas glandulares tipo VI e o grau de infestação da traça-do-tomateiro, e detectar as possíveis associações entre essas variáveis estudadas, em Lycopersicon esculentum, L. hirsutum f. glabratum e em $\mathrm{F}_{1}$ e $\mathrm{F}_{2}$ resultantes do cruzamento interespecífico.

\section{Material e Métodos}

Analisou-se o conteúdo de 2-tridecanona, a densidade de tricomas glandulares tipo VI e o grau de infestação da traça-do-tomateiro nos progenitores 'Uco Plata' (L. esculentum), suscetível, e PI 134417 (L. hirsutum f. glabratum ), resistente, e em $\mathrm{F}_{1}$ e $\mathrm{F}_{2}$, cultivados no campo experimental da Universidade Nacional de Salta (Argentina), em outubro (1998)/março (1999). A temperatura média durante o período de cultivo foi de $25^{\circ} \mathrm{C}$ e a fotofase média, de 13,4 horas. O cultivo foi feito com o sistema de irrigação por gotejo. As avaliações foram realizadas durante os meses de novembro e dezembro, utilizando-se um número variável de plantas tanto dos parentais como de $\mathrm{F}_{1}$ e $\mathrm{F}_{2}$ (Tabela 1).

Realizou-se a extração de 2-tridecanona por maceração e agitação dos folíolos de uma folha, pesados previamente, com n-hexano. As determinações realizaram-se em folhas maduras totalmente desenvolvidas. As amostras foram filtradas em um sistema de filtração de solventes marca Supelco.

A determinação da concentração de 2-tridecanona realizou-se por cromatografia gasosa em um cromatógrafo Hewlett Packard modelo 5890 série II, com coluna capilar INNOWax $(0,25 \mathrm{~mm}$ de diâmetro interno, $0,25 \mu \mathrm{m}$ de espessura do filme e $30 \mathrm{~m}$ de comprimento), detetor de 
ionização da fornalha e um integrador Hewlett Packard 3395. O gás empregado foi o nitrogênio. Foram injetados $0,3 \mu \mathrm{L}$ da amostra na câmara de injeção a $250^{\circ} \mathrm{C}$. A análise foi conduzida isotermicamente com uma temperatura do forno de $125^{\circ} \mathrm{C}$. O detetor foi utilizado a $300^{\circ} \mathrm{C}$. Realizaram-se duas determinações por amostras médias. Os dados de concentração de 2-tridecanona foram expressos em ppm de peso fresco.

A densidade de tricomas glandulares tipo VI, na superfície adaxial e abaxial da folha, foi determinada como média do número de pêlos contados sob lupa (40x) em quatro campos tomados ao acaso. A determinação foi realizada em folhas jovens completamente desenvolvidas. Os dados de densidade (números de pêlos/campo) foram transformados em $\sqrt{\mathrm{y}}$ para posterior análise.

A quantificação do grau de infestação dos insetos nas plantas adultas foi realizada seguindo uma escala de 0 a 4 sendo: 0 , sem galerias; 1 , início de galerias menores de $1 \mathrm{~mm}$ de comprimento; 2 , pequenas galerias com mais de $1 \mathrm{~mm}$ de comprimento; 3 , galerias grandes na folha infestada; 4, generalização de galerias em folhas adjacentes (Gray et al., 1999). Realizaram-se estimativas de herança ampla do grau de infestação com a traça por meio de relações entre variâncias, segundo Allard (1967):

$\mathrm{H}=\left(\mathrm{s}_{\mathrm{p}}^{2}-\sigma_{\mathrm{E}}^{2}\right) / \sigma_{\mathrm{p}}^{2}$,

$\sigma_{\mathrm{E}}^{2}=\left(\sigma_{\mathrm{p} 1}^{2}+\sigma_{\mathrm{p} 2}^{2}+\sigma_{\mathrm{F} 1}^{2}\right) / 3$

$\sigma_{\mathrm{p}}^{2}=$ variância fenotípica $=\sigma_{\mathrm{F} 2}^{2}$,

$\sigma^{2}=$ variância ambiental.

Utilizou-se o teste $\mathrm{t}$ de Student para comparar valores médios de 2-tridecanona, densidade de pêlos glandulares e grau de infestação nas populações progenitoras e progênies $\mathrm{F}_{1}$ e $\mathrm{F}_{2}$.

A associação entre o nível de 2-tridecanona, a densidade de tricomas glandulares tipo VI e o grau de infestação da traça-do-tomateiro determinou-se mediante uma análise de correlações simples.

\section{Resultados e Discussão}

A concentração média de 2-tridecanona detectada na população de PI 134417 foi significativamente maior que em 'Uco Plata' $(p<0,01)$. Seu valor foi 24 vezes superior ao do progenitor suscetível (Tabela 1). Esta diferença é maior que a detectada por Maluf et al. (1997), que encontraram em PI 134417 uma concentração de 2-tridecanona sete vezes maior que em L. esculentum. Fery \& Kennedy (1987) encontraram diferenças até setenta vezes maiores em PI 134417. Estas discrepâncias entre os resultados podiam ser explicadas pelos diferentes métodos de extração e quantificação do metabólito. Existe evidência sobre a influência da fotofase na concentração de 2-tridecanona (Kennedy et al., 1981); dessa forma, as diferenças também poderiam ser explicadas pelas distintas épocas de cultivo. Ao contrário da cultivar 'Uco Plata', PI 134417 apresentou maior variabilidade na concentração de 2-tridecanona (Tabela 1).

Os valores observados nas populações experimentais $F_{1}$ e $F_{2}$ mostram claramente como as médias se aproximam da média de 'Uco Plata', confirmando a hipótese de recessividade do caráter proposta por Kennedy (1986). Este autor propõe um modelo de herança de três genes recessivos. Por outro lado, o resultado em $F_{1}$ não concorda com os de Maluf et al. (1997) que detectaram em $F_{1}$ uma concentração de 2-tridecanona intermediária entre os parentais suscetível e resistente.

Do total de indivíduos da $\mathrm{F}_{2}$ analisados, somente três manifestaram um alto conteúdo de 2-tridecanona; no entanto, as concentrações observadas não superam o valor da média aritmética dos progenitores. Estas plantas resultaram estéreis.

Tabela 1. Concentração de 2-tridecanona (com base no peso fresco), grau de infestação da traça-do-tomateiro (escala de 0 a 4) e densidade de tricomas glandulares tipo VI em folhas de tomateiro suscetível (cultivar Uco Plata), resistente (linhagem PI 134417) e suas progênies $\mathrm{F}_{1}$ e $\mathrm{F}_{2}$, cultivado em condições de campo. Salta, Argentina, 1998/1999.

\begin{tabular}{|c|c|c|c|c|c|c|c|c|}
\hline \multirow[t]{2}{*}{ Tratamento } & \multirow[t]{2}{*}{$\begin{array}{l}\text { № de } \\
\text { plantas }\end{array}$} & \multirow[t]{2}{*}{$\begin{array}{l}\text { 2-tridecanona } \\
\qquad(\mathrm{ppm})\end{array}$} & \multirow[t]{2}{*}{$\begin{array}{l}\text { № de } \\
\text { plantas }\end{array}$} & \multirow[t]{2}{*}{$\begin{array}{c}\text { Grau de } \\
\text { infestação }\end{array}$} & \multirow[t]{2}{*}{$\begin{array}{l}\text { № de } \\
\text { plantas }\end{array}$} & \multicolumn{3}{|c|}{$\begin{array}{c}\text { Densidade de tricomas tipo VI } \\
\text { (no de pêlos/campo) }\end{array}$} \\
\hline & & & & & & Adaxial & Abaxial & Total \\
\hline 'Uco Plata' & 10 & $0,491 \pm 0,158$ & 14 & $3,7 \pm 0,46$ & 9 & $6,6 \pm 0,2$ & $4,3 \pm 0,5$ & $5,6 \pm 0,3$ \\
\hline PI 134417 & 11 & $11,746 \pm 3,173$ & 19 & $1,1 \pm 0,60$ & 20 & $5,7 \pm 0,3$ & $5,5 \pm 0,5$ & $5,6 \pm 0,5$ \\
\hline $\mathrm{F}_{1}$ & 13 & $0,933 \pm 0,302$ & 15 & $2,0 \pm 0,88$ & 12 & $6,8 \pm 0,5$ & $5,6 \pm 0,6$ & $6,3 \pm 0,4$ \\
\hline $\mathrm{F}_{2}$ & 69 & $1,382 \pm 1,080$ & 94 & $1,9 \pm 1,20$ & 107 & $5,9 \pm 0,3$ & $6,3 \pm 0,9$ & $5,9 \pm 0,3$ \\
\hline
\end{tabular}


$\mathrm{Na}$ análise de tricomas glandulares tipo VI, foram detectadas diferenças significativas, porém no limite de significação na densidade de pêlos da face adaxial e abaxial em ambas as matrizes ( $<<0,05$ em 'Uco Plata' e $p<0,05$ em PI 134417), sendo maior na superfície adaxial da folha. Com relação à densidade em $F_{2}$, não se detectaram diferenças significativas entre as duas superfícies $(p>0,05)$. Somente em $F_{1}$ a diferença foi altamente significativa $(p<0,01)$.

Verificou-se que a distribuição dos pêlos não é homogênea, sendo as áreas próximas às nervuras as que possuem maior densidade.

Comparando os valores médios de densidade de tricomas tipo VI entre o progenitor suscetível e o resistente, não se detectaram diferenças significativas $(\mathrm{p}>0,05)$, o que confirma dados de Snyder \& Carter (1984), em relação à abundância deste tipo de pêlo glandular em L. esculentum como também em L. hirsutum.

Considerando-se exclusivamente a superfície adaxial da folha, também não foram detectadas diferenças significativas ( $p>0,05)$; por outro lado, foram detectadas diferenças entre 'Uco Plata' e PI 134417 no tocante à densidade de tricomas na superfície abaxial $(p<0,05)$.

O grau de infestação médio da traça-do-tomateiro em 'Uco Plata' foi significativamente superior ao observado em PI $134417(\mathrm{p}<0,01)$. As médias de $\mathrm{F}_{1} \mathrm{e}$ $\mathrm{F}_{2}$ foram similares entre si e próximas ao valor médio aritmético entre ambos os parentais, situação que poderia explicar-se por um efeito médio gênico aditivo.

Estimou-se uma herança ampla de $72 \%$.

O coeficiente de correlação $(r=-0,061)$ entre a concentração de 2-tridecanona e a densidade total de tricomas tipo VI não foi significativo $(\mathrm{p}>0,05)$.

Embora a linhagem resistente e a cultivar suscetível tenham apresentado a mesma densidade deste tipo de tricoma, a diferença significativa entre a concentração de 2-tridecanona nas duas linhagens indica que a presença de pêlos glandulares não implica necessariamente a síntese deste metabólito. Portanto, sugere-se que o modelo de herança destes dois caracteres seja diferente.

O coeficiente de correlação $(r=-0,518)$ entre o grau de infestação da traça-do-tomateiro e a densidade total de tricomas tipo VI não foi significativo $(\mathrm{p}>0,05)$.
O coficiente de correlação simples estimado entre a concentração de 2-tridecanona e o grau de infestação da traça-do-tomateiro $\left(r=-0,286^{* *}\right)$ indica que entre estas duas variáveis existe uma leve mas altamente significativa associação negativa $(\mathrm{p}<0,01)$. O coeficiente de correlação estimado coincide com o obtido por Gray et al. (1999) $(\mathrm{r}=-0,326)$, mesmo quando neste último trabalho utilizou-se outro método de análise do nível de 2-tridecanona, razão pela qual confirmar-se-ia a fraca associação entre estas duas variáveis. A resistência do tomate à traça-do-tomateiro pode ser explicada parcialmente $\left(\mathrm{R}^{2}=8,17 \%\right)$ pelo nível de concentração de 2-tridecanona. Algum outro mecanismo de resistência adicional, independente da 2-tridecanona, estaria provavelmente envolvido na resistência a esta praga.

\section{Conclusões}

1. A presença de 2-tridecanona somente explica parcialmente a resistência do tomate à traça-do-tomateiro.

2. Os modelos de herança dos caracteres densidade de tricomas glandulares tipo VI e concentração de 2-tridecanona são diferentes.

3. A densidade de tricomas glandulares tipo VI é independente do grau de infestação.

\section{Referências}

ALLARD, R. W. Principios de la mejora genética de las plantas. Barcelona : Omega, 1967. 498 p.

EIGENBRODE, S. D.; TRUMBLE, J. T. Antibiosis to beet armyworm (Spodoptera exigua) in Lycopersicon accessions. Horticultural Science, Alexandria, v. 28, n. 9, p. 932-934, 1993.

FERY, R.; KENNEDY, G. G. Genetic analysis of 2-tridecanone concentration, leaf trichome characteristics and tobacco hornworm resistance in tomato. American Society for Horticultural Science Journal, Alexandria, v. 112, n. 5, p. 886-891, 1987.

GIUSTOLIN, T. A.; VENDRAMIM, J. D. Efeito dos aleloquímicos 2-tridecanona e 2-undecanona na biologia de Tuta absoluta Meyrick. Anais da Sociedade Entomológica do Brasil, Londrina, v. 25, n. 3, p. 417422, 1996 
GRAY, L.; COLLAVINO, G.; GILARDON, E.; HERNANDEZ, C.; OLSEN, A.; SIMÓN, G. Heredabilidad de la resistencia a la polilla del tomate (Tuta absoluta Meyrick) y su correlación genética con caracteres de calidad, en descendencias de cruzas interespecíficas del género Lycopersicon. Investigación Agraria. Producción y Protección Vegetales, Madrid, v. 14, n. 3, p. 445-451, 1999.

JUVIK, J. A.; STEVENS, M. A. Physiological mechanisms of host plant resistance in the genus Lycopersicon to Heliothis zea and Spodoptera exigua: two insect pests of cultivated tomato. American Society for Horticultural Science Journal, Alexandria, v. 107, n. 6, p. 1065-1669, 1982.

JUVIK, J.; BABKA, B.; TIMMERMANN, E. Influence of trichome exudates from species of Lycopersicon on oviposition behavior of Heliothis zea Boddie. Journal of Chemical Ecology, New York, v. 14, n. 4, p. 1261-1287, 1988.

KENNEDY, G. G. Consequences of modifying biochemically mediated insect resistance in Lycopersicon species. Washington : American Chemical Society, 1986. p. 130-140. (Symposium Series, 296).

KENNEDY, G. G.; FARRAR JUNIOR, R.; KASHYAP, R. K. 2-tridecanone-glandular trichome mediated insect resistance in tomato: effect on parasitoids and predators of Heliothis zea. Washington : American Chemical Society, 1991. p. 150-165. (Symposium Series, 449).

KENNEDY; G. G.; YAMAMOTO, R. T.; DIMOCK, M. B.; WILLAMS, W. G.; BORDNER, J. Effect of the daylength and light intensity on 2-tridecanone levels and resistance in Lycopersicon hirsutum f. glabratum to Manduca sexta. Journal of Chemical Ecology, New York, v. 7, n. 4, p. 707-716, 1981
LUCKNER, M. Secondary metabolism in microorganisms, plants and animals. Berlin : Springer, 1989. $563 \mathrm{p}$.

MALUF, W. R.; BARBOSA, L.; SANTA CECILIA, L. C. 2-tridecanone mediated mechanism of resistance to the South American tomato pinworm Scrobipalpuloides absoluta Meyrick (Lepidoptera-Gelechiidae) in Lycopersicon spp. Euphytica, Dordrecht, v. 93, p. 189-194, 1997.

MAXWELL, F. G.; JENNINGS, P. Breeding plants resistant to insects. New York : J. Wiley, 1980. 682 p.

ROSENTHAL, G. A.; BERENBAUM, M. Herbivores: their interactions with secondary plant metabolites. San Diego : Academic, 1992. 477 p.

SARAVIA, E.; COLOMBO, N.; BENAVENT, J. M. Resistencia a la polilla del tomate: estado actual de la selección. Sociedad Argentina de Olericultura Boletín Hortícola, Mendoza, v. 3, n. 4, p. 13-21, 1984.

SNYDER, J.; CARTER, C. Trichomes on leaves of Lycopersicon hirsutum, L. esculentum and their hybrids. Euphytica, Dordrecht, v. 34, p. 53-64, 1984.

WILLIAMS, G.; KENNEDY, G. G.; YAMAMOTO, J. D.; THACKER, J.; BORDNER, J. 2-tridecanone: a naturally occurring insecticide from the wild tomato Lycopersicon hirsutum f. glabratum. Science, Washington, v. 207, p. 888-889, 1980.

WINK, M. Plant breeding importance of plant secondary metabolites for protection against pathogens and herbivores. Theoretical Applied Genetics, Berlin, v. 75, p. 225-233, 1988. 